\title{
ATAD3 and endoplasmic reticulum to mitochondria connection: A main actor and interaction regarding pathogenesis
}

\author{
Denis Rousseau* \\ LBFA-INSERM U1055, Grenoble Alpes University, CS 40700, 38000 Grenoble, France
}

\begin{abstract}
Metabolic fluxes between mitochondria and endoplasmic reticulum appear today of first importance in normal and pathologic cellular physiology sustaining important regulation processes involved in mitochondrial biogenesis, homeostasis and stress responses. The basis of these interrelations is well described in yeast, the mechanism involved in higher eukaryotes and mammals is unknown. Here will be to present and emphasize on the role of a newly discovered protein, ATAD3, that may represent a molecular bridge between these compartments and essential link for associated exchanging processes.
\end{abstract}

\section{Introduction}

Mitochondria appeared in early eukaryotes, allowing thus a great multiplication of the cellular energetic power and opening gates to higher eukaryotes evolution [1].

Until human, mitochondria improved in their architecture, specificity (associated to cell differentiation), regulation pathways, biogenesis, renewal and elimination processes (mitophagy). All these progresses have allowed, step by step, a much better and sophisticated production and use of this main cellular energy source. To improve cellular efficiency, which depends on energy supply, it was needed to ameliorate mitochondria functioning and specification, as good examples with neurones (and synaptic mitochondria), muscles, spermatozoids or oocytes. More in details, mitochondria are responsible, in addition to their main ATP-producing function, of several other processes involved in diverse cellular functions. Mitochondria are thus essential for (i) lipogenesis, and reversely for beta-oxidation, (ii) membrane biogenesis and plasticity (like in producing phosphatidylcholine for plasma membrane, or sphingosine for myelinisation), (iii) steroids synthesis (especially in gonads or cortico-surrenal glands) and of course in (iiii) apoptosis processes, calcium homeostasis and signalization. Therefore, all the different mitochondrial activities are fully integrated to the whole cellular metabolism. This integration takes place especially through functional interactions with the main mitochondria partner which is the endoplasmic reticulum (ER) [2-5]. Indeed, mitochondrial biogenesis regulation is essentially supported by to the tight metabolic relations with the ER compartment, as well as their morphology, the produced reactive oxygen species, autophagy, apoptotic processes and calcium homeostasis [6,7]. These tight molecular connections between ER and mitochondria involve the MAM (Mitochondrial Associated Membranes) from one side and specific anchoring systems from mitochondrial side. Mitochondrial functions are therefore closely linked to their intercommunication with the ER, in both senses, showing how ER and mitochondria depend of each other and how a miss connection will impact both mitochondria and ER physiology [8-11]. These interactions are therefore involved in mitochondrial biogenesis and mitochondrial mass regulation according to the cell physiology and fate, like along tissue regeneration and aging processes.

Because of the main roles of mitochondria in cellular metabolism, mitochondrial dysfunctions can be responsible of very diverse diseases, severe and even rare, that can affect all organs. Therefore, it comes easy to understand how Endoplasmic Reticulum (ER)-mitochondria interactions can be potentially involved in certain pathologies. As central actors of cellular metabolism, the deficient physiology of mitochondria consecutively initiates various and numerous pathologies including neuropathies, myopathies like cardiopathies and ataxia, diabetes and cancers. Involved also in steroidogenesis, mitochondrial miss of function can also cause hormonal disorders and sterility. Then, any disorders in ER-M interactions may cause mitochondrial defects and related pathologies.

\section{Neuropathies and ER-M contact sites}

Mitochondria are of course the main cellular source of ATP, produced by the coupled activities of the Krebs cycle, the respiration chain and the F1F0 ATP synthase. Also, mitochondria are the site for de novo lipid synthesis, from Krebs cycle's citrate and Acetyl $\mathrm{CoA}$ "leakage" and lipid storage, as for lipid consumption by the $\beta$-oxidation. Mitochondria are therefore the crosstalk centre for bioenergetic regulations. For this reason, and because of their role in lipid metabolism, any mitochondrial miss function may have a direct impact on cell capacity to ensure their functions. Then it is not surprising to discover that many pathologies like myo- and neuro-

${ }^{\star}$ Correspondence to: Denis Rousseau, Applied and Fundamental Bioenergetic laboratory INSERM U1055, Bat B Joseph Fourier University, BP53 38041 Grenoble Cedex, France, Tel: (33)04 766356 00; Fax: (33)04 765142 18; E-mail: Denis.Rousseau@univ-grenoble-alpes.fr.

Received: February 25, 2019; Accepted: March 11, 2019; Published: March 18 2019 
pathies are mitochondria-linked and involve ER-M interaction defects $[7,12]$.

A significant disease, of modern times, Alzeimer disease, involves demyelination and degeneration of hippocampal and cortical neurons associated with intracellular accumulation of phosphorylated Tau and extracellular accumulation of $\beta$-amyloid peptides. The genetic causes can be either mutations of the precursor protein or of Presenilin which is involved in the processing of amyloid peptides and is enriched at ER-M contact sites [13]. In both patients and animal models, an increase of ER-M contacts was observed, associated with increase calcium flux and phospholipid biosynthesis $[14,15]$. Among the several hypothesis attempting to explain the pathogenesis, mitochondria dysfunctions have been shown recently to induce activation of sphingomyelinase, by oxidative stress and $\mathrm{H}_{2} \mathrm{O}_{2}$ over-production, provoking there and consecutively a demyelination process [16]. However, other hypothesis also exists to explain how and why ER-M contact sites and mitochondrial activities can be involved in the disease, but defective or abnormal ER-M interrelations can be clearly a cause for the disease [12].

Similarly, Parkinson disease, which is characterized by the degeneration of dopaminergic neurons, involves modifications of ER-M contact sites, implicating a-synuclein, DJ-1, Parkin, Mfn2 and PINK [17-20], and implying also BECN1 during mitophagy [21]. a-synuclein is found abundant at ER-M contact sites and its mutation induces a parkinsonism associated to decreased ER-M contact sites and of phospholipid synthesis. Parkin mutations are the main cause for Parkinson disease as Parkin, as PINK and BECN1 which are involved in mitophagy as well as in mitochondrial biogenesis, fission and transport. All these processes occur at ER-M contact sites. Indeed, increased contacts were observed in fibroblasts from patient with Parkin mutations so we can guess how much the disturbance of these sites could be linked to Parkinson disease [22,23].

Similarly, ER-M dysfunctions are also revealed in Amyotrophic lateral schlerosis and Huntington disease as in neuroinflammation $[7,12]$. As another example of ER-M dysfunction in neuropathies is a more recent case report on SERAC1 mutation related to MEGDEL syndrome $[24,25]$. This lipase-domain containing protein localizes at ER-M contact sites and may act by remodelling phosphatidylglycerol.

Finally, the best-case study to point again the importance of ER-M contacts in neuronal pathophysiology is MFN2 (Mitofusine 2). This mitochondrial protein involved in ER-M tethering is, under mutated form, responsible of the Charcot-Marie-Tooth neuropathic disease type 2A [26]. MFN2 contributes mainly in ER-M contact sites by creating a GTPase-dependent structure involving coiled-coil domain and supra-structure oligomerization that are implicated in mitochondrial fusion and ER-M interaction. Mutations in the GTPase, coiled-coil or conserved R3 domains induce severe neuropathies.

Mitochondria are also organelles involved in lipid and phospholipid synthesis like for cardiolipin, phosphatidyl Ethanolamine, phosphatidyl Serine, phosphatidyl Choline as for sphingomyelin synthesis, and for that, the ER is providing the required lipid precursors to the mitochondria [27-29]. The ER provides also mitochondria with glycosphingolipids for which the transfer may occurs also at MAM [30]. Therefore, any disturbance in the lipid fluxes between ER and mitochondria may have an important effect on lipid constitution/ equilibrium within the cell and the mitochondria and may impact drastically mitochondrial and cell activities. Concerning glial cells, a deficient lipid metabolism of mitochondria may contribute also in demyelination diseases or other unclassified neuropathies like in disease involving membrane dysfunction.

\section{Myopathies and ER-M contact sites}

As other high energy consuming organs, the muscles, heart and skeletal muscles, can also suffer intensively from mitochondrial missfunctions. Therefore, improper ER-M contact sites in muscles could be the cause of certain myopathies.

During muscle contraction, the ryanodine receptor permits the transfer of calcium from the ER (sarcoplasmic reticulum) to the mitochondria, to increase ATP production. The ryanodine receptor is also involved during muscle differentiation. Therefore, it is not surprising that disturbed ryanodine receptor functioning is related to myopathies. Indeed, ryanodine receptor mutation were observed in patients with central core disease and malignant hyperthermia [31,32]. Theses mutations induce alteration of calcium signalling and damaged mitochondria.

Calcium regulation is also modified in many muscular dystrophies [33]. As mitochondria play a significant role in calcium signalling and balancing, by an interplay with the endoplasmic reticulum involving the MAM [34], it is not so surprising to observe that a mutation of a regulator for the mitochondrial calcium uniporter induces pathological features like mitochondrial myopathies [35]. Then, targeting ER-M contact sites related to calcium balancing may be a solution to improve muscle function of patients $[33,36]$.

We guess that other muscular diseases will be found to reside in a miss functioning of ER-M contact sites but since this research area is still new, cause-effect relations between ER-M dysfunction and myopathies are still under investigations.

If we do not know today if these regulations are the cause or the consequence of the associated diseases, ER-M contact sites seem however to be of first importance in different and various neurological and muscle disorders.

\section{Obesity, diabetes, hepatic diseases and ER-M contact sites}

ER-M contact sites have been shown to be modulated by diets or nutritional transitions [37], and especially in the liver [38], and they are considered today as metabolic sensor site. Adaptations may occur during the switch between glucose oxidation and lipid oxidation, which depends of nutrition. Therefore, it is not surprising to observe that ER-M sites react to nutrition signals by the action of ER-stress system [39].

Therefore, metabolic diseases are also linked to mitochondrial activities and therefore to ER-M contact sites status. Obesity and diabetes have been also shown to involve ER-M contact sites regulations [40-42], and ER-stress activations were observed in several models for obesity and diabetes as well as in humans, and in different organs like the liver, the adipose tissue and skeletal muscle [43-45]. Reorganization of ER-M contact sites was correlated there with mitochondrial calcium overload, reduced oxidative capacities and increased oxidative stress. Also, molecules reducing ER-stress ameliorate the physiology of obese mice and humans $[46,47]$. The same observations were made in liver, in alcoholic and non-alcoholic diseases where ER-M contact sites are regulated and where active molecules can also modulate ER-M contact sites and produce beneficial effects [38].

\section{Sterility and ER-M contact sites}

Mitochondria are also main actors in steroidogenesis $[48,49]$. This role is first based on ER-M contact sites that promotes a cholesterol 
flux between ER and mitochondria where steroidogenesis takes place [50]. The transport of cholesterol is the rate-limiting step of steroidogenesis. It was demonstrated that the cholesterol transport activity, which depends of protein synthesis, is ensured by the protein StAR (steroidogenic acute regulatory protein), at ER-M contact sites, and that hormonal stimulation promotes both the cholesterol transport and the number of contacts [51]. StAR localizes in mitochondria, at the outer mitochondrial membrane and is associated to partner in the inner mitochondrial membrane and matrix protein [50]. Mutation in StAR gene cause congenital lipoid adrenal hyperplasia [52], a disease characterized by defective steroidogenesis. Mutations in any of the proteins involved in the cholesterol transport process may have a strong impact in steroid production, like for testosterone production and might induce therefore reduced fertility.

\section{Cancer and ER-M contact sites}

Many cancers are associated with increased cholesterol flux and ER-M contact sites. A one marker of breast cancer is TSPO (Translocator Protein), which belong to the cholesterol transport complex [53]. In other cancers, involving PML-driven malignant transformation, it has been observed that PML (promyelocytic leukaemia) partially localizes to ER-M contact site [54] and modulates Akt-dependent IP3R phosphorylation by the action of phosphatase 2A. The phosphorylation state of IP3R determines the amount of calcium driven from ER to mitochondria and is involved in apoptotic processes.

PTEN is another oncogene that can modulate Akt/IP3R signalling at the ER-M contact sites and that links cancer development and ER-M status [55]. Indeed, mTORC2 can localize to ER-M contact sites in growth factor stimulated cells in order to sense nutrient availability and metabolism. There, mTOTRC2 promotes the interaction between Grp75 and IP3R, regulates ATP production and is associated with increased phosphorylation of IP3R by Akt.

The famous tumour-suppressor protein $\mathrm{p}^{53}$ has also been reported to localize at ER-M contact sites and influence calcium signalling during cell stress [56]. Since most cancers have one or two mutated $\mathrm{p}^{53}$, which induce resistance to treatments, overexpression of SERCA or MCU can overcome this phenotype and sensitize the tumour cells to treatments [57].

Another example of improper ER-M contact sites involvement in cancer is the case study of $\mathrm{Bcl}-2$. It has been shown recently that $\mathrm{Bcl}-$ 2 inhibitor (ABT737) reverses cisplatin resistance by regulating ER-M calcium signalling [58]. Indeed, ABT737 treatment of ovarian cancer cells increases ER-M contact sites and increases cisplatin induced apoptosis. ER-M contact sites appears therefore as targets for anticancer therapy.

$\mathrm{Mfn} 2$ is also a cancer-associated gene. If overexpression of Mfn2 have been related to poor prognostic in gastric cancer [59], experiments have proved that Mfn2 presents tumour suppressor activity [60]. Both results are not contradictive, as Mfn2 overexpression in cancer can be of a mutated form, like for $\mathrm{p} 53$ in many tumour types.

Finally, TpM (trichioplein/mitostatin), a cytoskeleton binding protein under expressed in breast, prostate and bladder cancers was shown to play a role at ER-M contact sites and calcium signalling by interacting with MFN2 [61].

To conclude about ER-M contact sites and pathogenesis is to add finally that Ischemy-reperfusion contexts also implicate ER-M contact sites regulation, and molecules that can decrease ER-M contacts at reperfusion can protect cardiomyocytes against injuries and improve the success of heart vessels surgeries [62].

In yeast, the molecular organization of ER-M contact sites are today well described and called ERMES [5,9]. They involve Mdm1 Mdm10 Mdm12 Mdm34 and Mdm32.

However, in higher eukaryotes, where cholesterol takes place rather than ergosterol in yeast, and where mitochondrial mobility becomes tubulin-based, the protein complex involved in contact sites is still not known precisely but may involve a recently discovered protein, ATAD3 (ATPase family AAA Domain-containing protein 3).

\section{ATAD3 and ER-M contact sites}

The "mitochondrial mass" and its regulation is today a new concept in Cell Biology, Physiology and Medicine, and we are now considering that the mitochondrial "patrimoine" is a highly dynamic system that evolve all lifelong. Also, these regulations involve necessarily ER-M contact sites.

In higher eukaryotes, mitochondria can adapt, or not, at short, mid and long terms. Short terms adaptations are linked to short term signalizations, like post-translational regulations, while mid and long terms regulations concern translational and transcriptional levels. Increased feeding for example, or increased brain and muscular activities will induce mitochondrial biogenesis, mitochondrial mass increase, and this occurs at different regulation level and all along cell and body lives. At birth for example, the mitochondrial mass is developing a lot with breathing start, and thereafter at all phases and places involving cellular proliferation and differentiation (as the specific mitochondrial proteomic "editing" that occurs upon differentiation).

At the opposite, pathologies, aging, inactivity or nutritional starvation involved a general stress-response process that will lead to mitochondrial mass reduction and mitophagy.

The endoplasmic reticulum is in fact the first and major "associate" for the mitochondrial biogenesis. The ER provides mitochondria with several essential biomolecules like phosphatidyl-serine, cholesterol, phosphatidic acid, calcium, iron, zinc, amino-acids and probably nuclear tRNA and neo-synthesised, nuclear-encoded proteins $[8,29,63]$. Today we believe therefore that ER-M contact sites, via cholesterol raft and imported proteins system are crucial for mitochondrial biogenesis/ renewal [27].

The newly identified ATAD3 might be a mark of this steps for mitochondrial mass adaptation and ER-M interactions improvements along evolution.

ATAD3 is encoded by an immediate vital gene, necessary for development as soon as embryonic implantation stage [64], when the embryo starts to produce its "own", zygotic, mitochondria for first time. Along evolution, ATAD3 appears after yeast, in higher and pluricellular eukaryotic organisms, notably when cholesterol starts to take a place in membrane constitution and metabolism.

ATAD3 is an ATPase located in the inner mitochondrial membrane and is fully ubiquitous [65-67]. Then, its role can be important, essential, in all organs. Even if inserted through the inner mitochondrial membrane, ATAD3 is however suspected to interact with the outer membrane too, as even with endoplasmic reticulum-located partners. In more details ATAD3 has been shown to interact molecularly with Mfn2 and Drp1 [68], and functionally, as Drp1 and ATAD3 work in concert to drive mitochondrial biogenesis and fragmentation $[69,70]$. 
Also, ATAD3 interacts with WASF3 and GRP78 and this interaction may be involved during cancerogenesis [71]. Having its ATPase domain in the matrix [72], ATAD3 can constitute a bioenergetic sensor able to create a physical link-docking with the ER depending of ATP synthesis $[65,69]$. ATAD3 is also involved in stress-responses [71] and since ATAD3 interacts with $\mathrm{S} 100 \mathrm{~B}$ in a calcium-dependent manner, ATAD3 is also concerned by calcium signalling and regulation [69].

ATAD3 gene appeared as a single gene in pluricellular organisms, until rodents. In primates surprisingly, two others homologous genes appeared, probably by duplication. The three $A T A D 3$ genes, called therefore $A T A D 3 A, B$ and $C$ (where $A T A D 3 A$ is the ancestral form of $A T A D 3$ gene), present significant molecular differences (especially ATAD3C that does not possess large $\mathrm{N}$ - and C-terminal part) [73]. ATAD3A and ATAD3B were only studied yet, and the functional and molecular differences between both is especially the relative abundance and the tissue-specific expression differences. ATAD3A is expressed in all organs studied until now, and is expressed all life long, in embryo and adult. ATAD3B itself is expressed specifically in embryonic cells, in adult pituitary gland, and notably in tumours $[74,67,73]$. These differences are related to gene promoter particularities. Also, other shorter isoforms, produced by alternative splicing and translation, present specific patterns of expression [73].

Other differences between ATAD3A and ATAD3B are related to their phosphorylation sites and membrane structural insertion, but none of these particularities have today been explained [75].

If the precise function of ATAD3 is still not known today, the sure is that its expression level monitors mitochondrial biogenesis, as to be vital at very early stages of animal development. The first work concluding this is the ATAD3 Knock Down in D. melanogaster and C. elegans $[76,77]$ and the ATAD3 Knock Out in mouse [64].

In C. elegans, ATAD3-siRNA-based K.D. induces an arrest in the development, at L1 larvae stage. Indeed, the ATAD3 K.D. larva fed themselves normally but failed to accumulate lipids in their adipocytelike intestinal tissue. The authors showed indeed that the pre-required mitochondrial biogenesis does not occur efficiently in these cells, avoiding proper lipids storage [77].

In mouse, more recently, it has been shown that ATAD3 K.O. induces an early arrest of embryo development, at day 4 [64]. ATAD3/- early embryos appeared unable to develop their trophoblast and to implant onto the uterine mucosa. This phase corresponds to the first zygotic mitochondrial biogenesis. Even if ATAD3 is vital, the ATAD3+/- mice are viable under conventional breeding and did not show evident signs for any deficiencies. However, we may believe that allelic compensation may not function in all the situations and that haplo-insufficiencies could be revealed.

The most recent studies have lightened up a new and very interesting hypothesis developed by Papadopoulos and colleagues and deeply analysed [78-80]. They observed that ATAD3 may function as a part of a cholesterol-transporter, transferring cholesterol from the endoplasmic reticulum into the mitochondria, especially to provide lipids for mitochondrial-based steroids synthesis. We hypothesize ourselves that ATAD3 can contribute to drive the import of some nuclear-encoded mitochondrial proteins [70]. In fact, both hypotheses can fit and be joined together since we believe that RE exports (lipids and proteins), which support mitochondrial biogenesis, could occur as lipid-raft systems $[81,27,80]$.

Whatever hypothesis, we are today at a time to be certain that ATAD3 insures a major function in mitochondrial biogenesis like in correct proliferation and differentiation, by its contribution to ER-M contact sites and related transfers.

As a strong demonstration for this, recent studies have highlighted the major role of ATAD 3 and the impact of its miss of function. Indeed, single or bi-allelic ATAD3 mutation or deletion have been reported this and last years to induce dramatic neuro- and myopathies.

\section{ATAD3 and myopathies/neuropathies}

As expected, researches from the last two years highlighted this important role of ATAD3 in mitochondrial status and pathogenesis. Three important studies have described that ATAD3A/B mutations are associated with severe neuro-myopathies in humans [82-84]. Lupski's team identified a recurrent de novo $A T A D 3 A$ mutation in few unrelated individuals with a phenotype of developmental delay, hypotonia, optic atrophy, axonal neuropathy, and hypertrophic cardiomyopathy, as seen with MFN2, OPA1, DNM1L and STAT2 mutations [84]. They described two families with biallelic mutations of $A T A D 3 A$ and biallelic deletions issued from a nonallelic homologous recombination between $A T A D 3 A$ and $A T A D 3 B$ and ATAD3C. They found that these mutations induce in drosophila a dramatic decrease of mitochondrial mass, a modified mitochondrial morphology and an increased autophagy. Fibroblasts from patient exhibited also increased mitophagy. The same team reported these years a new set of pathological cases that links again ATAD3 mutations and other diseases for which no molecular diagnosis has been yet achieved as a clinical genomic diagnosis [82] The team of $\mathrm{H}$. Tyynismaa also described and linked ATAD 3 mutations to myopathies as spastic paraplegia [83]. They identified a dominantly inherited heterozygous mutation in $A T A D 3 A$ in an individual with hereditary spastic paraplegia and axonal neuropathy and his children with dyskinetic cerebral palsy, both diseases installed in childhood. They also showed that overexpression of the mutant ATAD3A induces fragmentation of the mitochondrial network in patient fibroblasts and neurons derived from their pluripotent stem cells. Mutations in ATAD3A is observed here also to be dominantly inherited. Also, more recently, a third study elucidated the genetic basis of cerebellar atrophy linked to ATAD3A/B deletion [85], stressing how much ATAD3 mutation can be deleterious.

In all case studies, the mutation affects the Walker A motif, responsible for ATP binding in the AAA module of ATAD3A. This mutation is believed to act as a dominant-negative because ATAD3 proteins may function as hexamers and mutant can compete negatively in the constitution of fully active polymers.

$A T A D 3 A$ variations/mutations represent therefore an additional link between mitochondrial dynamics and recognizable neurological and muscular syndromes, as expected. This finding extends therefore the group of mitochondrial inner membrane AAA proteins associated with spasticity.

\section{ATAD3 and Sterility}

Since ATAD3 is involved in ER-M contact sites and cholesterol transport, it is not surprising to find that ATAD3 controls steroids production in adrenocortical and Leydig cells $[79,78]$. Therefore, is to hypothesize that ATAD3 miss function/mutation can be the cause of certain infertility in males and females. Investigation of ATAD3 status in patient suffering of infecundity could be an interesting program of research.

\section{ATAD3 and cancer}

More importantly is the involvement of ATAD3 in cancer occurrence and development. Indeed, ATAD3 studies started with the 
observation of its overexpression in cancer [86] and that ATAD3 gene is a target gene of c-MYC [87]. This observation has been made in head and neck cancers, as in lung, uterine, glial, prostate and breast cancers [74,88-90]. Furthermore, ATAD3A/B expression correlates with chemoresistance and radioresistance of the tumours [74,91,92] as well as in metastasis activities through molecular interaction with GRP78 and modulation of WASF3 function [71]. Moreover, diminishing ATAD3A/B expression levels induces reversion of the transformed phenotype of glioma in vitro [74]. Therefore, decreasing ATAD3 activity is definitively a pertinent target for reversion of transformed phenotypes and limitation of tumour growth and invasion. Also, ATAD3 expression level detection on biopsies is a potential indicator for tumour classification and prognosis at hospital [75].

Before to conclude is to add, as we have seen before, that ER-M miss-regulations are involved in metabolic diseases like obesity, diabetes, or in fertility. It is therefore very attractive to see if any of these diseases can be cause by ATAD3 miss of function.

\section{Conclusion}

Along evolution, mitochondria physiology improved, and especially regarding the tight and important relation with the ER that became more and more complex and regulated.

Indeed, ER-M contact sites may support a coupled functioning between mtDNA control (replication/transcription/translation), translation and import of nuclear-encoded mitochondrial proteins, lipid fluxes and bioenergetic level. Therefore, an involvement of ATAD3 as a new importing raft system might be a good hypothetical model that could explain and support all these mechanisms.

\section{Reference}

1. Rousseau D (2016) An overview on the phylogeny of aerobic metabolism and about the preponderance of mitochondrial functions in evolution. J Genet Genome Res 3: 027.

2. Copeland DE, Dalton AJ (1959) An association between mitochondria and the endoplasmic reticulum in cells of the pseudobranch gland of a teleost. $J$ Biophys Biochem Cytol 5: 393-396. [Crossref]

3. Robertson JD (1960) The molecular structure and contact relationships of cell membranes. Prog Biophys Mol Biol 10: 343-418 [Crossref]

4. de Brito OM, Scorrano L (2008) Mitofusin 2 tethers endoplasmic reticulum to mitochondria. Nature 456: 605-610. [Crossref]

5. Eisenberg-Bord M, Shai N, Schuldiner M, Bohnert M (2016) A Tether Is a Tether Is a Tether: Tethering at Membrane Contact Sites. Dev Cell 39: 395-409. [Crossref]

6. Rizzuto R, Pinton P, Carrington W, Fay FS, Fogarty KE, et al. (1998) Close contacts with the endoplasmic reticulum as determinants of mitochondrial $\mathrm{Ca} 2+$ responses. Science 280: 1763-1766. [Crossref]

7. Rodriguez-Arribas M, Yakhine-Diop SM, Pedro JM, Gomez-Suaga P, Gomez-Sanchez R, et al. (2017) Mitochondria-Associated Membranes (MAMs): Overview and Its Role in Parkinson's Disease. Mol Neurobiol 54: 6287-6303. [Crossref]

8. Vance JE (1990) Phospholipid synthesis in a membrane fraction associated with mitochondria. J Biol Chem 265: 7248-7256. [Crossref]

9. Kornmann B, Walter P (2010) ERMES-mediated ER-mitochondria contacts: molecular hubs for the regulation of mitochondrial biology. J Cell Sci 123: 1389-1393. [Crossref]

10. Elbaz-Alon Y (2017) Mitochondria-organelle contact sites: the plot thickens. Biochem Soc Trans 45: 477-488. [Crossref]

11. Eisenberg-Bord M, Schuldiner M (2017) Mitochatting - If only we could be a fly on the cell wall. Biochim Biophys Acta 1864:1469-1480. [Crossref]

12. Erpapazoglou Z, Mouton-Liger F, Corti O (2017) From dysfunctional endoplasmic reticulummitochondria coupling to neurodegeneration. Neurochem Int 109: 171-183. [Crossref]

13. Naj AC, Schellenberg GD (2017) Genomic variants, genes, and pathways of Alzheimer's disease: An overview. Am J Med Genet B Neuropsychiatr Genet 174: 5-26. [Crossref]
14. Area-Gomez E, Del Carmen Lara Castillo M, Tambini MD, Guardia-Laguarta C, de Groof AJ, et al. (2012) Upregulated function of mitochondria-associated ER membranes in Alzheimer disease. EMBO J 31: 4106-4123. [Crossref]

15. Filadi R, Greotti E, Turacchio G, Luini A, Pozzan T, et al. (2016) Presenilin 2 Modulates Endoplasmic Reticulum-Mitochondria Coupling by Tuning the Antagonistic Effect of Mitofusin 2. Cell Rep 15: 2226-2238. [Crossref]

16. Klosinski LP, Yao J, Yin F, Fonteh AN, Harrington MG, et al. (2015) White Matter Lipids as a Ketogenic Fuel Supply in Aging Female Brain: Implications for Alzheimer's Disease. EBioMedicine 2: 1888-1904. [Crossref]

17. Koyano F, Okatsu K, Kosako H, Tamura Y, Go E, et al. (2014) Ubiquitin is phosphorylated by PINK1 to activate parkin. Nature 510: 162-166. [Crossref]

18. Chen Y, Dorn GW $2^{\text {nd }}$ (2013) PINK1-phosphorylated mitofusin 2 is a Parkin receptor for culling damaged mitochondria. Science 340: 471-475. [Crossref]

19. Guardia-Laguarta C, Area-Gomez E, Rub C, Liu Y, Magrane J, et al. (2014) $\alpha$-Synuclein is localized to mitochondria-associated ER membranes. J Neurosci 34 : 249-259. [Crossref]

20. Ottolini D, Calì T, Negro A, Brini M (2013) The Parkinson disease-related protein DJ-1 counteracts mitochondrial impairment induced by the tumour suppressor protein p53 by enhancing endoplasmic reticulum-mitochondria tethering. Hum Mol Genet 22 2152-2168. [Crossref]

21. Gelmetti V, De Rosa P, Torosantucci L, Marini ES, Romagnoli A, et al. (2017) PINK1 and BECN1 relocalize at mitochondria-associated membranes during mitophagy and promote ER-mitochondria tethering and autophagosome formation. Autophagy 13: 654-669. [Crossref]

22. Celardo I, Costa AC, Lehmann S, Jones C, Wood N, et al. (2016) Mitofusin-mediated ER stress triggers neurodegeneration in pink1/parkin models of Parkinson's disease. Cell Death Dis 7: e2271. [Crossref]

23. Lesage S, Drouet V, Majounie E, Deramecourt V, Jacoupy M, et al. (2016) Loss of VPS13C Function in Autosomal-Recessive Parkinsonism Causes Mitochondrial Dysfunction and Increases PINK1/Parkin-Dependent Mitophagy. Am J Hum Genet 98: 500-513. [Crossref]

24. Unal U, Uzgul RK, Yucel D, Yalnızoglu D, Tokatli A, et al. (2015) Two Turkish siblings with MEGDEL syndrome due to novel SERAC1 gene mutation. Turk J Pediatr 57: 388-393. [Crossref]

25. Wortmann SB, Vaz FM, Gardeitchik T, Vissers LE, Renkema GH, et al. (2012) Mutations in the phospholipid remodeling gene SERAC1 impair mitochondrial function and intracellular cholesterol trafficking and cause dystonia and deafness. Nat Genet 44 :797-802. [Crossref]

26. Feely SM, Laura M, Siskind CE, Sottile S, Davis M, et al. (2011) MFN2 mutations cause severe phenotypes in most patients with CMT2A. Neurology 76: 1690-1696. [Crossref]

27. Schlattner U, Tokarska-Schlattner M, Rousseau D, Boissan M, Mannella C, et al. (2014) Mitochondrial cardiolipin/phospholipid trafficking: the role of membrane contact site complexes and lipid transfer proteins. Chem Phys Lipids 179: 32-41. [Crossref]

28. Osman C, Voelker DR, Langer T (2011) Making heads or tails of phospholipids in mitochondria. J Cell Biol. 192: 7-16. [Crossref]

29. Voelker DR (2009) Genetic and biochemical analysis of non-vesicular lipid traffic. Annu Rev Biochem 78: 827-856. [Crossref]

30. Ardail D, Popa I, Bodennec J, Louisot P, Schmitt D, et al. (2003) The mitochondriaassociated endoplasmic-reticulum subcompartment (MAM fraction) of rat liver contains highly active sphingolipid-specific glycosyltransferases. Biochem $\mathrm{J}$ 371: 1013-1019. [Crossref]

31. Treves S, Jungbluth H, Muntoni F, Zorzato F (2008) Congenital muscle disorders with cores: the ryanodine receptor calcium channel paradigm. Curr Opin Pharmacol 8: 319326. [Crossref]

32. Rosenberg H, Davis M, James D, Pollock N, Stowell K (2007) Malignant hyperthermia Orphanet J Rare Dis 2: 21. [Crossref]

33. Vallejo-Illarramendi A, Toral-Ojeda I, Aldanondo G, Lopez de Munain A (2014) Dysregulation of calcium homeostasis in muscular dystrophies. Expert Rev Mol Med 16: e16. [Crossref]

34. Rizzuto R, Marchi S, Bonora M, Aguiari P, Bononi A, et al. (2009) Ca (2+) transfer from the ER to mitochondria: when, how and why. Biochim Biophys Acta 1787: 1342 1351. [Crossref] 
35. Logan CV, Szabadkai G, Sharpe JA, Parry DA, Torelli S, et al. (2014) Loss-of-function mutations in MICU1 cause a brain and muscle disorder linked to primary alterations in mitochondrial calcium signalling. Nat Genet 46: 188-193. [Crossref]

36. Goonasekera SA, Davis J, Kwong JQ, Accornero F, Wei-LaPierre L, et al. (2014) Enhanced $\mathrm{Ca}^{2+}$ influx from STIM1-Orail induces muscle pathology in mouse models of muscular dystrophy. Hum Mol Genet 23: 3706-3715. [Crossref]

37. Theurey P, Rieusset J (2017) Mitochondria-Associated Membranes Response to Nutrient Availability and Role in Metabolic Diseases. Trends Endocrinol Metab 28: 32-45. [Crossref]

38. Rieusset J (2017) Endoplasmic reticulum-mitochondria calcium signalling in hepatic metabolic diseases. Biochim Biophys Acta 1864: 865-876. [Crossref]

39. Schroder M (2008) Endoplasmic reticulum stress responses. Cell Mol Life Sci 65: 862894. [Crossref]

40. Rieusset J (2015) Contribution of mitochondria and endoplasmic reticulum dysfunction in insulin resistance: Distinct or interrelated roles? Diabetes Metab 41: 358-368. [Crossref]

41. Arruda AP, Pers BM, Parlakgul G, Guney E, Inouye K, et al. (2014) Chronic enrichment of hepatic endoplasmic reticulum-mitochondria contact leads to mitochondrial dysfunction in obesity. Nat Med 20: 1427-35. [Crossref]

42. Koliaki C, Roden M (2013) Hepatic energy metabolism in human diabetes mellitus, obesity and non-alcoholic fatty liver disease. Mol Cell Endocrinol 379: 35-42. [Crossref]

43. Gregor MF, Yang L, Fabbrini E, Mohammed BS, Eagon JC, et al. (2009) Klein S. Endoplasmic reticulum stress is reduced in tissues of obese subjects after weight loss. Diabetes 58: 693-700. [Crossref]

44. Boden G, Duan X, Homko C, Molina EJ, Song W, et al. (2008) Increase in endoplasmic reticulum stress-related proteins and genes in adipose tissue of obese, insulin-resistant individuals. Diabetes 57: 2438-2444. [Crossref]

45. Ozcan U, Cao Q, Yilmaz E, Lee AH, Iwakoshi NN, et al. (2004) Endoplasmic reticulum stress links obesity, insulin action, and type 2 diabetes. Science 306: 457-61. [Crossref]

46. Ozcan U, Yilmaz E, Ozcan L, Furuhashi M, Vaillancourt E, et al. (2006) Chemical chaperones reduce ER stress and restore glucose homeostasis in a mouse model of type 2 diabetes. Science 313: 1137-1140. [Crossref]

47. Kars M, Yang L, Gregor MF, Mohammed BS, Pietka TA, et al. (2010) Tauroursodeoxycholic Acid may improve liver and muscle but not adipose tissue insulin sensitivity in obese men and women. Diabetes 59: 1899-1905. [Crossref]

48. Poderoso C, Duarte A, Cooke M, Orlando U, Gottifredi V, et al. (2013) The spatial and temporal regulation of the hormonal signal. Role of mitochondria in the formation of a protein complex required for the activation of cholesterol transport and steroids synthesis. Mol Cell Endocrinol 371: 26-33 [Crossref]

49. Issop L, Rone MB, Papadopoulos V (2013) Organelle plasticity and interactions in cholesterol transport and steroid biosynthesis. Mol Cell Endocrinol 371: 34-46. [Crossref]

50. Elustondo P, Martin LA, Karten B (2017) Mitochondrial cholesterol import. Biochim Biophys Acta 1862: 90-101. [Crossref]

51. Clark BJ, Wells J, King SR, Stocco DM (1994) The purification, cloning, and expression of a novel luteinizing hormone-induced mitochondrial protein in MA-10 mouse Leydig tumour cells. Characterization of the steroidogenic acute regulatory protein (StAR). $J$ Biol Chem 269: 28314-28322. [Crossref]

52. Miller WL (1997) Congenital lipoid adrenal hyperplasia: the human gene knockout for the steroidogenic acute regulatory protein. J Mol Endocrinol 19: 227-240. [Crossref]

53. Mukherjee S, Das SK (2012) Translocator protein (TSPO) in breast cancer. Curr Mol Med 12: 443-57. [Crossref]

54. Pinton P, Giorgi C, Pandolf PP (2011) The role of PML in the control of apoptotic cell fate: a new key player at ER-mitochondria sites. Cell Death Differ 18: 1450-1456. [Crossref]

55. Betz C, Stracka D, Prescianotto-Baschong C, Frieden M, Demaurex N, et al. (2013) Feature Article: mTOR complex 2-Akt signaling at mitochondria-associated endoplasmic reticulum membranes (MAM) regulates mitochondrial physiology. Proc Natl Acad Sci U S A 110: 12526-34. [Crossref]

56. Bittremieux M, Bultynck G (2015) p53 and $\mathrm{Ca}(2+)$ signalling from the endoplasmic reticulum: partners in anti-cancer therapies. Oncoscience 2: 233-238. [Crossref]

57. Giorgi C, Bonora M, Sorrentino G, Missiroli S, Poletti F, et al. (2015) p53 at the endoplasmic reticulum regulates apoptosis in a $\mathrm{Ca} 2+-$ dependent manner. Proc Natl Acad Sci U S A 112: 1779-84. [Crossref]
58. Xie Q, Su J, Jiao B, Shen L, Ma L, et al. (2016) ABT737 reverses cisplatin resistance by regulating ER-mitochondria $\mathrm{Ca} 2+$ signal transduction in human ovarian cancer cells. Int J Oncol 49: 2507-2519. [Crossref]

59. Fang CL, Sun DP, Chen HK, Lin CC, Hung ST, et al. (2017) Overexpression of Mitochondrial GTPase MFN2 Represents a Negative Prognostic Marker in Human Gastric Cancer and Its Inhibition Exerts Anti-Cancer Effects. $J$ Cancer 8: 1153-1161. [Crossref]

60. Xu K, Chen G, Li X, Wu X, Chang Z, et al. (2017) MFN2 suppresses cancer progression through inhibition of mTORC2/Akt signaling. Sci Rep 7: 41718 [Crossref]

61. Cerqua C, Anesti V, Pyakurel A, Liu D, Naon D, et al. (2010) Trichoplein/mitostatin regulates endoplasmic reticulum-mitochondria juxtaposition. EMBO Rep 11: 854-860. [Crossref]

62. Paillard M, Tubbs E, Thiebaut PA, Gomez L, Fauconnier J, et al. (2013) Depressing mitochondria-reticulum interactions protects cardiomyocytes from lethal hypoxiareoxygenation injury. Circulation 128: 1555-1565. [Crossref]

63. Gowher A, Smirnov A, Tarassov I, Entelis N (2013) Induced tRNA import into human mitochondria: implication of a host aminoacyl-tRNA-synthetase. PLoS One 8: e66228. [Crossref]

64. Goller T, Seibold UK, Kremmer E, Voos W, Kolanus W (2013) Atad3 function is essential for early post-implantation development in the mouse. PLoS One 8: e54799. [Crossref]

65. Bogenhagen DF, Rousseau D, Burke S (2008) The layered structure of human mitochondrial DNA nucleoids. J Biol Chem 283: 3665-75. [Crossref]

66. Lev S (2010) Non-vesicular lipid transport by lipid-transfer proteins and beyond. $\mathrm{Nat}$ Rev Mol Cell Biol 11: 739-750. [Crossref]

67. Merle N, Feraud O, Gilquin B, Hubstenberger A, Kieffer-Jacquinot S, et al. (2012) ATAD3B is a human embryonic stem cell specific mitochondrial protein, re-expressed in cancer cells, that functions as dominant negative for the ubiquitous ATAD3A. Mitochondrion 12: 441-488. [Crossref]

68. Chiang SF, Huang CY, Lin TY, Chiou SH, Chow KC (2012) An alternative import pathway of AIF to the mitochondria. Int J Mol Med 29: 365-372. [Crossref]

69. Gilquin B, Cannon BR, Hubstenberger A, Moulouel B, Falk E, et al. (2010) The calcium-dependent interaction between S100B and the mitochondrial AAA ATPase ATAD3A and the role of this complex in the cytoplasmic processing of ATAD3A. Mol Cell Biol 30: 2724-2736. [Crossref]

70. Li S, Bouzar C, Cottet-Rousselle C, Zagotta I, Lamarche F, et al. (2016) Resveratrol inhibits lipogenesis of 3T3-L1 and SGBS cells by inhibition of insulin signalling and mitochondrial mass increase. Biochim Biophys Acta 1857: 643-652. [Crossref]

71. Teng Y, Ren X, Li H, Shull A, Kim J, et al. (2016) Mitochondrial ATAD3A combines with GRP78 to regulate the WASF3 metastasis-promoting protein. Oncogene 35: 333 343. [Crossref]

72. Hubstenberger A, Merle N, Charton R, Brandolin G, Rousseau D (2010) Topological analysis of ATAD3A insertion in purified human mitochondria. J Bioenerg Biomembr 42: 143-150. [Crossref]

73. Li S, Lamarche F, Charton R, Delphin C, Gires O, et al. (2014) Expression analysis of ATAD3 isoforms in rodent and human cell lines and tissues. Gene 535: 60-69. [Crossref]

74. Hubstenberger A, Labourdette G, Baudier J, Rousseau D (2008) ATAD 3A and ATAD $3 \mathrm{~B}$ are distal $1 \mathrm{p}$-located genes differentially expressed in human glioma cell lines and present in vitro anti-oncogenic and chemoresistant properties. Exp Cell Res 314: 28702883. [Crossref]

75. Li S, Rousseau D (2012) ATAD3, a vital membrane bound mitochondrial ATPase involved in tumour progression. J Bioenerg Biomembr 44: 189-197. [Crossref]

76. Kamath RS, Ahringer J (2003) Genome-wide RNAi screening in Caenorhabditis elegans. Methods 30: 313-321 [Crossref]

77. Hoffmann M, Bellance N, Rossignol R, Koopman WJ, Willems PH, et al. (2009) C. elegans ATAD-3 is essential for mitochondrial activity and development. PLoS One 4: e7644. [Crossref]

78. Rone MB, Midzak AS, Issop L, Rammouz G, Jagannathan S, et al. (2012) Identification of a dynamic mitochondrial protein complex driving cholesterol import, trafficking, and metabolism to steroid hormones. Mol Endocrinol 26: 1868-1882. [Crossref]

79. Issop L, Fan J, Lee S, Rone MB, Basu K, et al. (2015) Mitochondria-associated membrane formation in hormone-stimulated Leydig cell steroidogenesis: role of ATAD3. Endocrinology 156: 334-345. [Crossref] 
80. Gerhold JM, Cansiz-Arda S, Lohmus M, Engberg O, Reyes A, et al. (2015) Human Mitochondrial DNA-Protein Complexes Attach to a Cholesterol-Rich Membrane Structure. Sci Rep 5: 15292. [Crossref]

81. He J, Cooper HM, Reyes A, Di Re M, Sembongi H, et al. (2012) Mitochondrial nucleoid interacting proteins support mitochondrial protein synthesis. Nucleic Acids Res 40: 6109-6121. [Crossref]

82. Eldomery MK, Coban-Akdemir Z, Harel T, Rosenfeld JA, Gambin T, et al. (2017) Lessons learned from additional research analyses of unsolved clinical exome cases. Genome Med 9: 26. [Crossref]

83. Cooper HM, Yang Y, Ylikallio E, Khairullin R, Woldegebriel R, et al. (2017) ATPasedeficient mitochondrial inner membrane protein ATAD3A disturbs mitochondrial dynamics in dominant hereditary spastic paraplegia. Hum Mol Genet 26:1432-1443. [Crossref]

84. Harel T, Yoon WH, Garone C, Gu S, Coban-Akdemir Z, et al. (2016) Recurrent De Novo and Biallelic Variation of ATAD3A, Encoding a Mitochondrial Membrane Protein, Results in Distinct Neurological Syndromes. Am J Hum Genet 99: 831-845. [Crossref]

85. Desai R, Frazier AE, Durigon R, Patel H, Jones AW, et al. (2017) ATAD3 gene cluster deletions cause cerebellar dysfunction associated with altered mitochondrial DNA and cholesterol metabolism. Brain 140:1595-1610. [Crossref]

86. Schaffrik M, Mack B, Matthias C, Rauch J, Gires O (2006) Molecular characterization of the tumour-associated antigen AAA-TOB3. Cell Mol Life Sci 63: 2162-2174 [Crossref]
87. Zeller KI, Jegga AG, Aronow BJ, O’Donnell KA, Dang CV (2003) An integrated database of genes responsive to the Myc oncogenic transcription factor: identification of direct genomic targets. Genome Biol 4: R69. [Crossref]

88. Fang HY, Chang CL, Hsu SH, Huang CY, Chiang SF, et al. (2010) ATPase family AAA domain-containing $3 \mathrm{~A}$ is a novel anti-apoptotic factor in lung adenocarcinoma cells. J Cell Sci 123: 1171-80. [Crossref]

89. Chen TC, Hung YC, Lin TY, Chang HW, Chiang IP, et al. (2011) Human papillomavirus infection and expression of ATPase family AAA domain containing $3 \mathrm{~A}$, a novel antiautophagy factor, in uterine cervical cancer. Int J Mol Med 28: 689-696. [Crossref]

90. Huang KH, Chow KC, Chang HW, Lin TY, Lee MC (2011) ATPase family AAA domain containing $3 \mathrm{~A}$ is an anti-apoptotic factor and a secretion regulator of PSA in prostate cancer. Int J Mol Med 28: 9-15. [Crossref]

91. You WC, Chiou SH, Huang CY, Chiang SF, Yang CL, et al. (2013) Mitochondrial protein ATPase family, AAA domain containing $3 \mathrm{~A}$ correlates with radioresistance in glioblastoma. Neuro Oncol 15: 1342-1352. [Crossref]

92. Ovaska K, Matarese F, Grote K, Charapitsa I, Cervera A, et al. (2013) Integrative analysis of deep sequencing data identifies estrogen receptor early response genes and links ATAD3B to poor survival in breast cancer. PLoS Comput Biol 9: e1003100. [Crossref]

Copyright: @2019 Rousseau D. This is an open-access article distributed under the terms of the Creative Commons Attribution License, which permits unrestricted use, distribution, and reproduction in any medium, provided the original author and source are credited. 